Published in final edited form as:

Anal Chem. 2019 July 16; 91(14): 9284-9291. doi:10.1021/acs.analchem.9b02261.

\title{
Native Mass Spectrometry of Antimicrobial Peptides in Lipid Nanodiscs Elucidates Complex Assembly
}

\author{
Lawrence R. Walker ${ }^{\dagger, 1}$, Elaine M. Marzluff ${ }^{\dagger, 2}$, Julia A. Townsend ${ }^{1}$, William C. Resager ${ }^{1}$, and \\ Michael T. Marty ${ }^{1,}{ }^{*}$ \\ ${ }^{1}$ Department of Chemistry and Biochemistry, The University of Arizona, Tucson, AZ 85721 \\ 2Department of Chemistry, Grinnell College, Grinnell, IA 50112
}

\begin{abstract}
Antimicrobial peptides (AMPs) are generally cationic and amphipathic peptides that show potential applications to combat the growing threat of antibiotic resistant infections. AMPs are known to interact with bacterial membranes, but their mechanisms of toxicity and selectivity are poorly understood, in part because it is challenging to characterize AMP oligomeric complexes within lipid bilayers. Here, we used native mass spectrometry (MS) to measure the stoichiometry of AMPs inserted into lipoprotein nanodiscs with different lipid components. Titrations of increasing peptide concentration and collisional activation experiments reveal that AMPs can exhibit a range of behaviors from non-specific incorporation into the nanodisc to formation of specific complexes. This new approach to characterizing formation of AMP complexes within lipid membranes will provide unique insights into AMP mechanisms.
\end{abstract}

\section{Graphical Abstract}

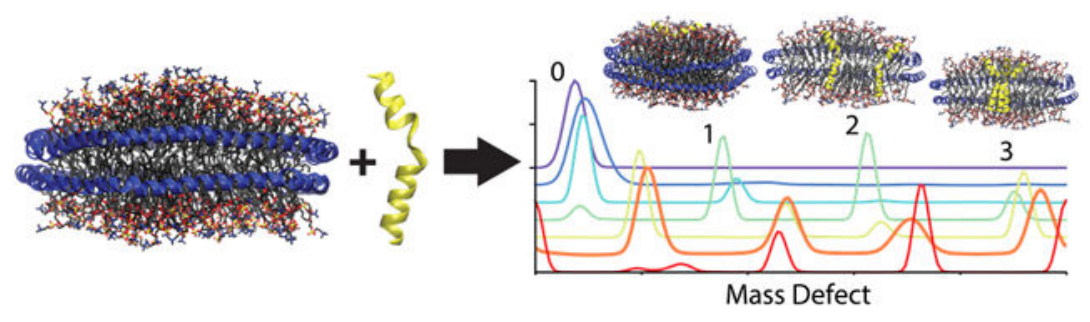

\section{Introduction}

Antimicrobial peptides (AMPs) are secreted by a variety of organisms as natural defenses against microbial pathogens. ${ }^{1}$ Generally cationic and amphipathic, AMPs often interact directly with bacterial membranes rather than specific protein targets. Because it may be

\footnotetext{
‘Corresponding Author mtmarty@email.arizona.edu.

$\dagger$ These authors contributed equally.

SUPPORTING INFORMATION

The Supporting Information is available free of charge on the ACS Publications website and contains supplemental figures and tables.

Notes

The authors declare no competing financial interests. UniDec software can be downloaded at: https://github.com/michaelmarty/

UniDec/releases. No unexpected or unusually high safety hazards were encountered in this study.
} 
harder (although not impossible ${ }^{2}$ ) for bacteria to evolve resistance to AMPs, they present a potential strategy to combat antibiotic resistant infections. However, the mechanisms of AMP toxicity and selectivity remain poorly understood, which creates challenges for development of AMPs as pharmaceuticals ${ }^{3}$ and as anti-biofilm materials. ${ }^{4}$

Although a common mechanism of AMP activity is formation of oligomeric pores, ${ }^{3}$ it is difficult to characterize the stoichiometry of AMP complexes within lipid membranes. ${ }^{5}$ Conventional techniques for measuring oligomeric states are complicated by several factors. The small size and chemical diversity of AMPs limit chemical cross-linking, and fluorescent labels may disrupt their interactions. The lipid bilayer complicates hydrodynamic methods such as analytical ultracentrifugation. Potential polydispersity of AMP complexes limits conventional structural biology tools. Conductance-based measurements can indirectly estimate oligomeric states of AMP but cannot observe complexes that do not form pores. ${ }^{6}$

Native mass spectrometry (MS) has emerged as an effective tool for characterizing stoichiometry of biomolecular complexes. ${ }^{7,8}$ Conventional native MS methods for studying membrane protein oligomeric states rely on using collision induced dissociation (CID) to eject membrane proteins from detergent micelles, ${ }^{9,10}$ which are unsuitable for AMP because the lipid bilayer is essential for complex formation and is thought to drive the pharmaceutical specificity. ${ }^{3}$

Russell and coworkers used liposomes to investigate AMP gramicidin A (GA), which is known to form dimers, with ion mobility-MS (IM-MS). However, isobutanol was required to disrupt the vesicles and extract the free peptide for electrospray ionization (ESI), which may disrupt AMP complexes. ${ }^{11-13}$ Klassen and coworkers co-incorporated GA into nanodiscs, ${ }^{14}$ which contain a lipid bilayer encircled by an amphipathic membrane scaffold protein (MSP), 15 and ionized the entire GA-nanodisc complex. Because the intact GA-nanodisc could not be resolved, CID was used to eject GA from the nanodisc for subsequent IM-MS. However, ejection of AMPs from bilayers may disrupt their stoichiometry, as evidenced by the mixture of GA monomers and dimers ejected from the nanodisc. Because complexes may be broken apart upon ejection, CID experiments must be carefully interpreted, as discussed below.

Here, we propose that high-resolution native MS of intact AMP-nanodisc complexes can reveal the stoichiometry of AMPs incorporated in lipid bilayers. Formation of oligomeric complexes can be inferred from the distribution of observed stoichiometries. For example, GA shows only even stoichiometries, indicating the formation of dimers. In contrast, melittin incorporates into membranes but shows no specific stoichiometries. LL-37 shows partial specificity for certain oligomeric states. Together, these data reveal that AMPs have different behaviors ranging from random incorporation into membranes to formation of specific oligomeric complexes. Native MS of intact nanodiscs provides a novel approach to quantifying oligomerization of peptides within membranes that will help elucidate the mechanisms of AMP complex assembly. 


\section{Methods}

\section{Materials and Nanodisc Assembly}

1,2-dimyristoyl-sn-glycero-3-phosphocholine (DMPC) and 1,2-dimyristoyl-sn-glycero-3phospho-(1'-rac-glycerol) (DMPG) lipids were purchased from Avanti Polar Lipids. LL-37 was purchased from Bachem. Gramicidin A was purchased from Alfa Aesar. Melittin was purchased from GenScript. Ammonium acetate, Amberlite XAD-2, and sodium cholate were purchased from Sigma Aldrich. Membrane scaffold protein MSP1D1(-) was expressed in $E$. coli and purified by immobilized metal affinity chromatography (IMAC) as previously described. ${ }^{16,17}$

Nanodiscs were assembled without peptides as previously described. ${ }^{16,17}$ Briefly, DMPC or DMPG lipids in chloroform were dried under nitrogen and solubilized in sodium cholate. MSP1D1(-) was added to the lipids, and detergent was removed by addition of Amberlite XAD-2 hydrophobic beads. Nanodiscs were purified using a Superose 6 Increase 10/300 column (GE Healthcare) with $0.2 \mathrm{M}$ ammonium acetate at $\mathrm{pH}$ 6.8. ${ }^{18}$

\section{Mass Spectrometry Sample Preparation}

Previously, Klassen and coworkers co-incorporated GA during the nanodisc self-assembly reaction. ${ }^{14}$ Here, we directly added peptides to the pre-formed nanodiscs. Purified nanodisc stocks were prepared at $2.5 \mu \mathrm{M}$ in $0.2 \mathrm{M}$ ammonium acetate (pH 6.8). Peptide stocks were prepared by dissolving a known mass of peptide in methanol and diluting to concentrations from $0.025-0.30 \mathrm{mM}$. Methanol was used because GA has poor solubility in water. Peptides were titrated at molar ratios of $0-18 / 1$ total peptide/nanodisc by adding peptide stocks directly to nanodisc stocks and incubating for a few minutes prior to analysis. Control experiments were performed to verify that the peptide/nanodisc mixture had reached equilibrium and that no time-dependent changes in incorporation were observed during analysis. The kinetics of peptide incorporation were generally too fast to observe in the roughly one minute required to manually mix the solutions, add the sample to the ESI needle, and begin collecting data. The final concentration of nanodiscs in solution was $2 \mu \mathrm{M}$, and final peptide concentrations ranged from $0-36 \mu \mathrm{M}$, which encompassed the minimum inhibitory concentrations measured previously. ${ }^{19-21}$ All solutions contained $13 \%$ methanol by volume, and controls with no peptides showed that nanodiscs were not perturbed by this methanol concentration. A stock solution of imidazole was prepared at $0.40 \mathrm{M}$ and adjusted to $\mathrm{pH} 6.8$ with acetic acid. ${ }^{22}$ To determine the optimal imidazole concentration, imidazole was titrated at a fixed ratio of peptide/nanodisc over a $0-48 \mathrm{mM}$ imidazole range. Except where noted, all experiments were performed at a final concentration of $25 \mathrm{mM}$ imidazole. Typical added volumes were $19 \mu \mathrm{L}$ nanodisc stock, $3 \mu \mathrm{L}$ peptide stock, and $1.5 \mu \mathrm{L}$ imidazole stock.

\section{Native Mass Spectrometry}

Native MS was performed using a Q-Exactive HF quadrupole-Orbitrap mass spectrometer with the Ultra-High Mass Range (UHMR) research modifications (Thermo Fisher Scientific) ${ }^{23}$ Instrumental parameters were used as previously described. ${ }^{16,22,24}$ Samples were ionized by nano-ESI in positive ion mode with a $1.1 \mathrm{kV}$ capillary voltage and $200{ }^{\circ} \mathrm{C}$ 
capillary temperature. For titrations, nanodiscs were analyzed from 2,000-25,000 $\mathrm{m} / \mathrm{z}$ at a resolution setting of 15,000 (a transient length of $32 \mathrm{~ms}$ ) and a trapping gas pressure setting of 7 (a high vacuum pressure of 233 nanobar). Nitrogen was used as the trapping/collision gas. To aid in desolvation, $50 \mathrm{~V}$ source fragmentation and $20 \mathrm{~V}$ in-source trapping energy were applied.

For CID experiments, the in-source trapping voltage was increased from 0-100 V or 0-140 $\mathrm{V}$ in 10 or $20 \mathrm{~V}$ increments with 1-minute acquisitions at each step. To detect dissociated peptides, nanodisc complexes were isolated in the quadrupole with an isolation range of $5,000-15,000 \mathrm{~m} / \mathrm{z}$ and dissociated by an additional $50 \mathrm{~V}$ in the HCD cell. Scans were collected from $500-20,000 \mathrm{~m} / \mathrm{z}$ at a resolution setting of 60,000 (a transient length of 128 $\mathrm{ms}$ ) and a trapping gas pressure setting of 3 or 5 (high vacuum levels of 102 or 168 nanobar, respectively).

For free peptides, peptide stocks were diluted to $25 \mu \mathrm{M}$ in ammonium acetate with imidazole, and MS was performed from $360-5,000 \mathrm{~m} / \mathrm{z}$ at a resolution setting of 60,000 and a trapping gas pressure setting of 3 . No activation was applied for analysis of free peptides. Control experiments were also performed by adding peptides to free MSP in ammonium acetate solution with no lipids. Because no MSP-peptide complexes were observed, we conclude that there are no peptide-MSP interactions and that peptide-nanodisc interactions are driven by the lipid bilayer.

\section{Deconvolution and Mass Defect Analysis}

Deconvolution of native mass spectra was performed using UniDec and MetaUniDec. ${ }^{16,24,25}$ Deconvolution settings for nanodiscs included a mass range of 20k-200k, a charge range of $5-25$, and a peak FWHM of 10. Both charge and mass smoothing were used with a width of 1, and the lipid mass (678 Da for DMPC or 667 Da for DMPG) was used as the mass difference. To sharpen the peaks, raw/centroid mode was used. UniDec employs a Bayesian deconvolution algorithm similar to the Richardson/Lucy algorithm. ${ }^{25}$ As part of the algorithm, peaks are deconvolved from their normal Gaussian, Lorentzian, or split Gaussian/ Lorentzian peak shapes into delta functions. Normally, the peak shape is convolved with the delta function outputs after deconvolution so that the deconvolved mass distributions reflect the peak shapes of the original spectra. Here, we used the raw outputs to take advantage of the improved resolution provided by deconvolution. Comparisons with the default reconvolved/profile mode showed no significant differences in peak positions or relative intensities.

Following deconvolution, we used macromolecular mass defect analysis in MetaUniDec to determine the number of peptides incorporated into nanodiscs. Mass defect analysis divides the measured mass by a reference mass and plots the remainder of the division, which we normalized between 0 and $1 .^{22,26}$ Here, we use the mass of the lipid as the reference mass. Mass defects are initially calculated for each deconvolved mass. Because nanodiscs with the same number of incorporated peptides but different number of lipids have the same mass defect, we summed the mass defects across all masses to generate the total mass defect distribution. Thus, each peak in the total mass defect distribution showed the summed intensity for all nanodiscs containing specific stoichiometries of incorporated peptides. 
To prepare titration and dissociation curves, we extracted the peak height for mass defect peaks. Because mass defect peaks had very similar peak widths, peak areas showed similar results but were less robust when partially overlapping peaks were present. Generally, mass defect peaks were unambiguous to assign to a specific number of incorporated peptides based on predicted mass defects (Table S1). In several cases, ambiguities were resolved through CID experiments. For example, the mass defect of LL-37 with 2 MSP belts and DMPG is 0.58 for 2 incorporated and 0.54 for 6 . CID was used to track products and infer the number incorporated from the dissociation and other observed stoichiometries in the titration series. Error bars are shown as the standard deviation of three trials, each with a different batch of nanodiscs. As previously observed, ${ }^{24}$ we found that the primary source of variation is between batches of nanodiscs.

\section{Results and Discussion}

\section{Method Development and Optimization}

We recently showed that the oligomeric state of membrane proteins can be measured in intact nanodiscs by native MS by carefully controlling ESI conditions. ${ }^{22}$ Here, we hypothesized that the stoichiometry of AMPs could be measured in intact nanodiscs, avoiding potential distortions by detergents, organic solvents, or CID. We tested this hypothesis using three AMPs: GA, melittin, and LL-37. Sequences and properties for each peptide are included in Table S1. Nanodiscs were formed using either DMPC or DMPG. DMPC is a zwitterionic lipid that models the generally neutral charge of eukaryotic plasma membranes, and DMPG is an anionic lipid that models the negative charge of bacterial membranes. ${ }^{27}$ Unlike membrane proteins, AMPs were added directly to "empty" nanodiscs (containing only lipid and MSP) prior to native MS, which mimics how AMPs interact with natural membranes. A schematic of the approach is shown in Figure 1.

Mass spectra of AMP nanodiscs are complex due to different numbers of peptides and lipids incorporated in the nanodiscs (Figure 1B). UniDec, a Bayesian deconvolution algorithm, was used to deconvolve $\mathrm{m} / \mathrm{z}$ spectra into mass distributions (Figure 1C). ${ }^{24}$ We determined the stoichiometry of incorporated AMPs using small shifts in the mass distributions measured by macromolecular mass defect analysis (Figure 1D). ${ }^{22,26}$ Mass defect analysis sums the intensity from masses that differ in number of lipids per nanodisc but contain the same number of incorporated peptides. Distinct peaks were observed for different numbers of peptides incorporated per nanodisc. We extracted and normalized the height of each summed mass defect peak to get the relative intensity of each peptide stoichiometry (Figure 1E). From the mass alone, we cannot distinguish between peptides embedded in the bilayer and peptides associated with the surface; we use the term incorporated to include both types of interaction.

To quantify AMP stoichiometries, we needed to ensure the normalized intensities reflected the true levels of AMP incorporation. Because the $\mathrm{m} / \mathrm{z}$ values were similar for all complexes, we assumed ionization and detection efficiencies were similar. Thus, we needed to ensure that AMP nanodiscs were uniformly stable during ionization and analysis. To optimize the ionization conditions, we employed imidazole as a charge reducing reagent, which stabilizes empty nanodiscs in positive ionization mode. ${ }^{22}$ By titrating the imidazole concentration at 
an intermediate level of peptide loading, we discovered that 20-40 $\mathrm{mM}$ imidazole significantly stabilizes AMP nanodiscs, leading to higher observed levels of AMPs in nanodiscs (Figure S1). In this respect, AMP nanodiscs behaved like empty nanodiscs and not like membrane protein nanodiscs. ${ }^{22}$ However, imidazole concentrations greater than 30 $\mathrm{mM}$ generally caused poorer resolution and lower signal/noise. Thus, we used $25 \mathrm{mM}$ imidazole to stabilize AMP nanodiscs for all subsequent experiments.

We next optimized the collision voltage applied in the injection flatapole to desolvate the nanodisc complexes. AMP stoichiometries were monitored as a function of collision voltage (Figure S2 \& S3). The source fragmentation voltage was also optimized to $50 \mathrm{~V}$ and had a limited impact on measured peptide incorporation. Although CID was observed with increasing voltage for some lipid and peptide combinations, the AMP stoichiometries were constant up to at least $20 \mathrm{~V}$. Thus, we limited the desolvation voltage to $0-20 \mathrm{~V}$ except when performing CID experiments. The combination of minimal CID and imidazole limits disruption of the complex, and we expect that nanodiscs are largely intact in the gas phase under these conditions.

\section{Gramicidin A}

To validate that stoichiometries observed by native MS reflected the solution-phase distribution, we investigated gramicidin $\mathrm{A}(\mathrm{GA})$, a 15 amino acid peptide with a neutral charge. GA is known to form dimers in membranes, ${ }^{28}$ and two distinct structural models have been proposed. ${ }^{29,30} \mathrm{In}$ ammonium acetate solution, we did not detect free GA peptide, likely due to poor solubility in the absence of nanodiscs.

Addition of GA to nanodiscs provided well-resolved spectra with several new peak series. Representative mass spectra, deconvolved mass spectra, and summed mass defects are shown in Figure 2, and representative mass defects as a function of mass and peptide concentration are shown in Figure S4. Interestingly, we observe an initial decrease in the mass of nanodiscs corresponding to the loss of around 20-25 lipids when only a 1.5:1 molar ratio of GA is added - before we see substantial incorporation of GA into nanodiscs.

However, it is not clear whether GA causes lower masses by solution-phase displacement of lipids or gas-phase destabilization of the nanodisc bilayer. A similar loss of 20-25 lipids is also observed with both melittin and LL-37.

Upon titration of GA into nanodiscs (Figure 2), we only observe incorporation of even stoichiometries of GA for both lipids. The absence of odd stoichiometries indicates that GA forms dimers, validating that native MS reflects the known stoichiometries within membranes. Because the distribution of dimers fits a binomial distribution at each concentration, the presence of 4 or more GA is likely caused by random incorporation of multiple dimers as opposed to formation of higher order oligomers. Slightly higher incorporation was observed for DMPC, but the similar levels of incorporation between DMPG and DMPC indicate that GA does not have a strong preference for lipid head groups, likely due to the lack of charged residues on GA.

In contrast with the intact mass analysis, CID is highly dependent on lipid head group. GA remains in DMPC nanodiscs during CID, and we did not observe ejected peptides (Figure 
S2B). Klassen and coworkers previously showed ejection of both dimers and monomers from nanodiscs with PC lipids, ${ }^{14}$ so the absence of ejected dimer here could be due to instrumental limitations. Specifically, Klassen's use of ion mobility to separate ejected dimer from the much more abundant ejected DMPC signal may be critical to having the dynamic range to observe the weak ejected dimer signal. On the other hand, GA is readily ejected from DMPG nanodiscs. Importantly, we observe DMPG nanodiscs containing 1 or 3 GA after activation, indicating ejection of monomers (Figure S2A). Thus, GA dimers can be disrupted with CID. When nanodiscs were selected in the quadrupole and activated, no ejected dimers were observed (Figure S5). Instead, we observed a strong signal from dissociated GA monomer with a small peak for a single bound DMPG. Because CID can distort the oligomeric states of AMPs in membranes, it must be carefully interpreted and is best used to support intact mass data.

\section{Melittin}

Melittin, the primary component of honey bee venom, is a 26 amino acid peptide with a net charge of +5 (Table S1). Melittin was initially thought to form tetramers, ${ }^{31,32}$ but subsequent evidence has shown that it has more complex interactions with lipid bilayers that remain poorly understood. ${ }^{19,27,33,34}$ At $25 \mu \mathrm{M}$, melittin was only detected as a monomer in ammonium acetate in the absence of nanodiscs (Figure S6). Representative mass defects for melittin titrated into DMPG nanodiscs are shown in Figure S7. Like GA, addition of melittin causes an initial decrease in the nanodisc mass, which occurs at molar ratios less than 3 melittin added per nanodisc and before we observe substantial incorporation of melittin (Figure 3). The mass does not continue to shift after the initial drop.

Comparing titrations with different lipids, significantly more melittin is incorporated into DMPG than DMPC nanodiscs (Figure 3), with as many as 7 melittin molecules incorporated into DMPG and only 4 for DMPC. Electrostatic interactions between the cationic melittin and the anionic DMPG likely drive the increased incorporation compared to zwitterionic DMPC. Some prior studies have also shown melittin to be more active in membranes with $\mathrm{PG},{ }^{19}$ but others have shown no preference. ${ }^{34}$

Although early studies suggested that melittin forms tetramers, ${ }^{31,32}$ the relative populations of melittin stoichiometries here do not suggest any specific oligomeric complexes. At each concentration, the ratios of each stoichiometry are reasonably modelled by a binomial distribution of monomers. The binomial nature of incorporation suggests that melittin does not favor any oligomeric states. Thus, melittin could be forming non-specific complexes and/or simply incorporating as monomers that are not interacting.

We investigated how melittin nanodiscs dissociate under CID. For both DMPC and DMPG nanodiscs, increasing collision voltages lead to progressive decrease in higher stoichiometries and increase in lower stoichiometries (Figure S3). Dissociation of DMPG nanodiscs isolated in the quadrupole only shows ejection of monomeric melittin bound to a small number of lipids (Figure S8). Dissociated melittin was not observed during dissociation of isolated DMPC nanodiscs, likely due to the high signal from dissociated DMPC and lower levels of incorporation. 
Because melittin oligomers could be disrupted by ejection from the nanodisc, we cannot rule out the presence of nonspecific oligomers based on the CID data. However, the lack of ejected oligomeric complexes (in contrast to LL-37 below) and the nonspecific incorporation into intact nanodiscs suggest that intermolecular interactions between melittin peptides are weak. The presence of lipids bound to ejected melittin does indicate that interactions with DMPG lipids are strong enough to survive ejection from the nanodisc, likely due to electrostatic interactions between the anionic lipid head group and cationic residues on melittin.

\section{LL-37}

LL-37 is a human cathelicidin with 37 amino acids and a net charge of +6 (Table S1). ${ }^{35}$ LL-37 is thought to form oligomers, but the precise forms and the effects of lipids are poorly understood. ${ }^{20,36,37}$ We observe that LL-37 in solution at $25 \mu \mathrm{M}$ shows a mixture of monomers, dimers, trimers, and tetramers (Figure S9). Upon titration of low LL-37 ratios into DMPC nanodiscs, only small amounts of LL-37 incorporation were observed, and LL-37 destabilized DMPC nanodiscs at ratios greater than 6/1 (Figure 4E). These results agree with previous work that showed LL-37 causing large scale disruptions of the cell membrane. ${ }^{38}$

In contrast, DMPG nanodiscs were stable as LL-37 was added (Figure 4). Representative mass defects are shown in Figure S10. Unlike melittin, LL-37 stoichiometries do not fit random binomial distributions or increase sequentially (Figure 4). Interestingly, DMPG nanodiscs with two LL-37 incorporated appear at lower concentrations than nanodiscs with only one LL-37 incorporated. These unusual results can be clearly seen in the raw data (Figure S11). We do not observe an appreciable level of 3 incorporated, but it instead jumps to 4 peptides/nanodisc. At higher ratios, incorporation shows preferences for 4, 5, and 6 peptides/nanodisc, but we did not observe any higher stoichiometries at 7 or above. These data suggest that LL-37 forms oligomeric complexes that prefer specific stoichiometries, with special preference for dimers, tetramers, pentamers, and hexamers. Future work will be required to model these complex equilibria, but LL-37 clearly forms complexes with partial specificity.

CID experiments provide further confirmation of complex formation. Collisional activation of LL-37 DMPG nanodiscs reveals two dissociation pathways: ejection of monomers and ejection of larger complexes. Like melittin and GA, monomeric LL-37 can be ejected from nanodiscs, which leads to the sequential decrease in signals from higher stoichiometries and increase in lower stoichiometries (Figure S3). For example, nanodiscs with three LL-37 incorporated are weakly populated at low collision voltage but become more abundant at higher collision voltages. Activation of isolated LL-37 nanodiscs shows ejection of monomeric LL-37 bound to several DMPG lipids (Figure S12). Interestingly, at low molar ratios, the intensity of nanodiscs with two LL-37 molecules decreases non-sequentiallydecreasing the intensity for two LL-37 without increasing the intensity for one (Figure S13). Because ejected dimers were not observed, it could be that the initial dissociation of a monomer catalyzes ejection of the remaining monomer. It could also be that ejected dimers are not stable and further dissociate into two monomers before they can be detected. 
At higher molar ratios, however, LL-37 can dissociate by ejection of larger complexes. Ejected trimers, tetramers, pentamers, and hexamers were directly detected bound to DMPG lipids, which is not observed with GA and melittin (Figure S14). These dissociated complexes may not represent the distribution of oligomeric states in the membrane, either due to loss of monomers or bias in stabilities and/or detection. For example, a significant amount of dissociated trimer is observed, but the intact mass only shows a small amount of nanodiscs with three peptides incorporated. Nevertheless, because these complexes remain intact after ejection from the nanodisc, we conclude that LL-37 has reasonably strong peptide-peptide and peptide-lipid interactions and forms oligomeric complexes in membranes.

\section{Conclusion}

These results demonstrate that native MS of intact nanodiscs provides a uniquely powerful approach to measuring stoichiometry of AMP complexes in lipid bilayers. By adding imidazole to stabilize nanodiscs and using minimal collisional activation, we can monitor the incorporation of AMPs into nanodiscs with different lipids and at increasing concentrations of AMPs. By measuring the distribution of incorporated stoichiometries, we can infer formation of oligomeric complexes in the membrane. Comparing stoichiometries between nanodisc containing different lipids shows how the lipid bilayer influences incorporation of AMPs. Although CID helped confirm complex formation with LL-37, disruption of complexes during CID complicates interpretation of dissociation data.

We found that GA forms specific dimer complexes but shows only minor preference for different lipid head groups. In contrast, melittin did not show formation of specific complexes but showed significantly higher incorporation into DMPG nanodiscs, which mimic bacterial membranes. Finally, LL-37 showed more complex lipid interactions and partial specificitiy for certain oligomeric states, including higher order oligomers.

Future work will explore the thermodynamics and kinetics of AMPs in nanodiscs more deeply, but this initial study demonstrates the potential of native MS to characterize the assembly and lipid interactions of AMP complexes in defined lipid bilayers, which has not previously been possible. Because lipid interactions are critical to AMP activity, our approach may provide mechanistic insights on how changes in the lipid bilayer lead to resistance, either by decreasing overall association of AMPs with the membrane or by disrupting formaiton of specific complexes within the membrane. Ultimately, we expect that simultaneously studying both AMP-membrane interactions and AMP complex formation will lead to new mechanistic insights into their toxicity and selectivity.

\section{Supplementary Material}

Refer to Web version on PubMed Central for supplementary material.

\section{ACKNOWLEDGMENTS}

The authors thank Maria Reinhardt-Szyba, Kyle Fort, and Alexander Makarov at Thermo Fisher Scientific for support on the UHMR Q-Exactive HF. The pMSP1D1 plasmid was a gift from Stephen Sligar (Addgene plasmid no. 20061). This work was funded by the Bisgrove Scholar Award from Science Foundation Arizona, the American 
Society for Mass Spectrometry Research Award, the National Science Foundation (CHE-1845230), and the National Institute of General Medical Sciences and National Institutes of Health (R35 GM128624) to MTM. The content is solely the responsibility of the authors and does not necessarily represent the official views of the NIH. The authors thank James Rohrbough for experimental contributions.

\section{ABBREVIATIONS}

$\begin{array}{ll}\text { AMP } & \text { Antimicrobial peptide } \\ \text { CID } & \text { collision induced dissociation } \\ \text { DMPC } & \text { dimyristoyl-phosphatidylcholine } \\ \text { DMPG } & \text { dimyristoyl-phosphatidylglycerol } \\ \text { GA } & \text { Gramicidin A } \\ \text { MS } & \text { mass spectrometry } \\ \text { MSP } & \text { membrane scaffold protein }\end{array}$

\section{REFERENCES}

1. Boto A; Pérez de la Lastra J; González C The Road from Host-Defense Peptides to a New Generation of Antimicrobial Drugs. Molecules 2018, 23, 311.

2. Joo HS; Fu CI; Otto M Bacterial strategies of resistance to antimicrobial peptides. Philos. Trans. R. Soc. Lond. B Biol. Sci 2016, 371.

3. Li J; Koh JJ; Liu S; Lakshminarayanan R; Verma CS; Beuerman RW Membrane Active Antimicrobial Peptides: Translating Mechanistic Insights to Design. Front. Neurosci 2017, 11, 73. [PubMed: 28261050]

4. Yasir M; Willcox MDP; Dutta D Action of Antimicrobial Peptides against Bacterial Biofilms. Materials (Basel, Switzerland) 2018, 11, E2468.

5. Ulmschneider JP; Ulmschneider MB Molecular Dynamics Simulations Are Redefining Our View of Peptides Interacting with Biological Membranes. Acc. Chem. Res 2018, 51, 1106-1116. [PubMed: 29667836]

6. Seydlová G; Sokol A; Lišková P; Konopásek I; Fišer R Daptomycin Pore Formation and Stoichiometry Depend on Membrane Potential of Target Membrane. Antimicrob. Agents Chemother 2019, 63, e01589-01518. [PubMed: 30323037]

7. Leney AC; Heck AJ Native Mass Spectrometry: What is in the Name? J. Am. Soc. Mass Spectrom 2017, 28, 5-13. [PubMed: 27909974]

8. Chandler SA; Benesch JLP Mass spectrometry beyond the native state. Curr. Opin. Chem. Biol 2018, 42, 130-137. [PubMed: 29288996]

9. Calabrese AN; Radford SE Mass spectrometry-enabled structural biology of membrane proteins. Methods 2018, 147, 187-205. [PubMed: 29510247]

10. Bolla JR; Agasid MT; Mehmood S; Robinson CV Membrane Protein-Lipid Interactions Probed Using Mass Spectrometry. Annu. Rev. Biochem 2019, DOI: 10.1146/annurevbiochem-013118-111508.

11. Patrick JW; Gamez RC; Russell DH Elucidation of Conformer Preferences for a Hydrophobic Antimicrobial Peptide by Vesicle Capture-Freeze-Drying: A Preparatory Method Coupled to Ion Mobility-Mass Spectrometry. Anal. Chem 2015, 87, 578-583. [PubMed: 25522119]

12. Patrick JW; Gamez RC; Russell DH The Influence of Lipid Bilayer Physicochemical Properties on Gramicidin A Conformer Preferences. Biophys. J 2016, 110, 1826-1835. [PubMed: 27119642]

13. Patrick JW; Zerfas B; Gao J; Russell DH Rapid capillary mixing experiments for the analysis of hydrophobic membrane complexes directly from aqueous lipid bilayer solutions. Analyst 2017, 142, 310-315. [PubMed: 27957567] 
14. Li J; Richards MR; Kitova EN; Klassen JS Delivering Transmembrane Peptide Complexes to the Gas Phase Using Nanodiscs and Electrospray Ionization. J. Am. Soc. Mass. Spectrom 2017, 28, 2054-2065. [PubMed: 28681358]

15. Bayburt TH; Grinkova YV; Sligar SG Self-assembly of discoidal phospholipid bilayer nanoparticles with membrane scaffold proteins. Nano Lett 2002, 2, 853-856.

16. Reid DJ; Keener JE; Wheeler AP; Zambrano DE; Diesing JM; Reinhardt-Szyba M; Makarov A; Marty MT Engineering Nanodisc Scaffold Proteins for Native Mass Spectrometry. Anal. Chem 2017, 89, 11189-11192. [PubMed: 29048874]

17. Ritchie TK; Grinkova YV; Bayburt TH; Denisov IG; Zolnerciks JK; Atkins WM; Sligar SG: Reconstitution of Membrane Proteins in Phospholipid Bilayer Nanodiscs In Methods Enzymol.; Nejat D, Ed.; Academic Press: San Diego, CA, 2009; Vol. 464; pp 211-231. [PubMed: 19903557]

18. Marty MT; Zhang H; Cui W; Blankenship RE; Gross ML; Sligar SG Native mass spectrometry characterization of intact nanodisc lipoprotein complexes. Anal. Chem 2012, 84, 8957-8960. [PubMed: 23061736]

19. Jamasbi E; Batinovic S; Sharples RA; Sani M-A; Robins-Browne RM; Wade JD; Separovic F; Hossain MA Melittin peptides exhibit different activity on different cells and model membranes. Amino Acids 2014, 46, 2759-2766. [PubMed: 25200682]

20. Bonucci A; Caldaroni E; Balducci E; Pogni R A Spectroscopic Study of the Aggregation State of the Human Antimicrobial Peptide LL-37 in Bacterial versus Host Cell Model Membranes. Biochemistry 2015, 54, 6760-6768. [PubMed: 26502164]

21. Wang F; Qin L; Pace CJ; Wong P; Malonis R; Gao J Solubilized Gramicidin A as Potential Systemic Antibiotics. ChemBioChem 2012, 13, 51-55. [PubMed: 22113881]

22. Keener JE; Zambrano DE; Zhang G; Zak CK; Reid DJ; Deodhar BS; Pemberton JE; Prell JS; Marty MT Chemical additives enable native mass spectrometry measurement of membrane protein oligomeric state within intact nanodiscs. J. Am. Chem. Soc 2019, 141, 1054-1061. [PubMed: 30586296]

23. van de Waterbeemd M; Fort KL; Boll D; Reinhardt-Szyba M; Routh A; Makarov A; Heck AJ High-fidelity mass analysis unveils heterogeneity in intact ribosomal particles. Nat. Methods 2017 , 14, 283-286. [PubMed: 28114288]

24. Reid DJ; Diesing JM; Miller MA; Perry SM; Wales JA; Montfort WR; Marty MT MetaUniDec: High-Throughput Deconvolution of Native Mass Spectra. J. Am. Soc. Mass Spectrom 2019, 30, 118-127. [PubMed: 29667162]

25. Marty MT; Baldwin AJ; Marklund EG; Hochberg GK; Benesch JL; Robinson CV Bayesian deconvolution of mass and ion mobility spectra: from binary interactions to polydisperse ensembles. Anal. Chem 2015, 87, 4370-4376. [PubMed: 25799115]

26. Marty MT; Hoi KK; Gault J; Robinson CV Probing the Lipid Annular Belt by Gas-Phase Dissociation of Membrane Proteins in Nanodiscs. Angew. Chem. Int. Ed. Engl 2016, 55, 550-554. [PubMed: 26594028]

27. Guha S; Ghimire J; Wu E; Wimley WC Mechanistic Landscape of Membrane-Permeabilizing Peptides. Chem. Rev 2019, DOI: 10.1021/acs.chemrev.8b00520.

28. Veatch W; Stryer L The dimeric nature of the gramicidin A transmembrane channel: conductance and fluorescence energy transfer studies of hybrid channels. J. Mol. Biol 1977, 113, 89-102. [PubMed: 69713]

29. Ketchem R; Hu W; Cross T High-resolution conformation of gramicidin A in a lipid bilayer by solid-state NMR. Science 1993, 261, 1457-1460. [PubMed: 7690158]

30. Höfer N; Aragão D; Caffrey M Crystallizing transmembrane peptides in lipidic mesophases. Biophys. J 2010, 99, L23-L25. [PubMed: 20682243]

31. Terwilliger TC; Eisenberg D The structure of melittin. II. Interpretation of the structure. J. Biol. Chem 1982, 257, 6016-6022. [PubMed: 7076662]

32. Tosteson MT; Tosteson DC The sting. Melittin forms channels in lipid bilayers. Biophys. J 1981, 36, 109-116. [PubMed: 6269667]

33. Yang Z; Choi H; Weisshaar JC Melittin-Induced Permeabilization, Re-sealing, and Repermeabilization of E. coli Membranes. Biophys. J 2018, 114, 368-379. [PubMed: 29401434] 
34. Sychev SV; Balandin SV; Panteleev PV; Barsukov LI; Ovchinnikova TV Lipid-dependent pore formation by antimicrobial peptides arenicin-2 and melittin demonstrated by their proton transfer activity. J. Pept. Sci 2015, 21, 71-76. [PubMed: 25522354]

35. Xhindoli D; Pacor S; Benincasa M; Scocchi M; Gennaro R; Tossi A The human cathelicidin LL-37 - A pore-forming antibacterial peptide and host-cell modulator. Biochim. Biophys. Acta 2016, 1858, 546-566. [PubMed: 26556394]

36. Sancho-Vaello E; François P; Bonetti E-J; Lilie H; Finger S; Gil-Ortiz F; Gil-Carton D; Zeth K Structural remodeling and oligomerization of human cathelicidin on membranes suggest fibril-like structures as active species. Sci. Rep 2017, 7, 15371. [PubMed: 29133814]

37. Dürr UHN; Sudheendra US; Ramamoorthy A LL-37, the only human member of the cathelicidin family of antimicrobial peptides. Biochim. Biophys. Acta 2006, 1758, 1408-1425. [PubMed: 16716248]

38. den Hertog AL; van Marle J; van Veen HA; Van’t Hof W; Bolscher JG; Veerman EC; Nieuw Amerongen AV Candidacidal effects of two antimicrobial peptides: histatin 5 causes small membrane defects, but LL-37 causes massive disruption of the cell membrane. Biochem. J 2005, 388, 689-695. [PubMed: 15707390] 


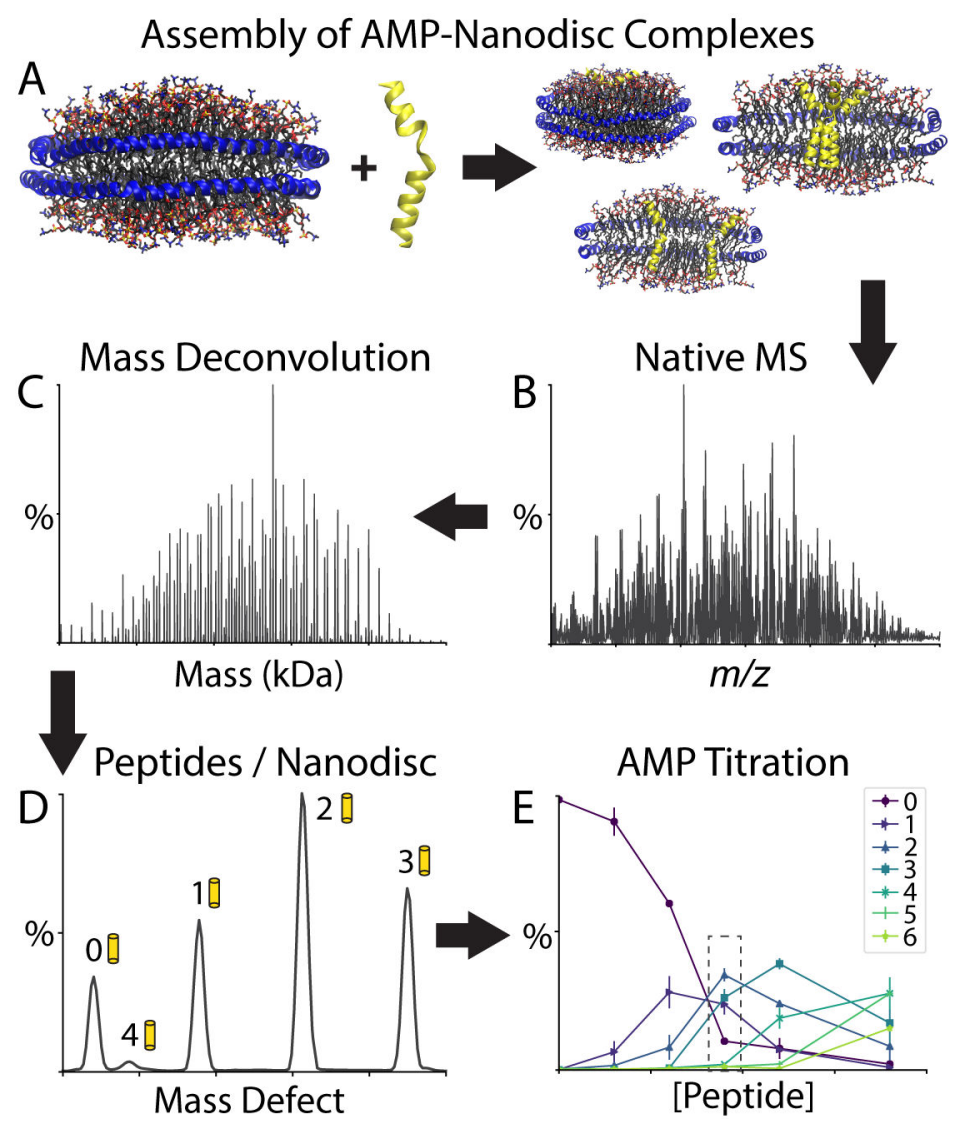

Figure 1.

Schematic of experimental approach. AMPs were added to nanodiscs (A) and the resulting mixture was analysed by native MS (B). Deconvolution revealed the mass distribution (C), and mass defect analysis was used to extract the total signals from nanodiscs containing different numbers of incorporated AMPs per nanodiscs (D) as a function of the total concentration of peptide added (E). The distribution in (D) corresponds to the dashed box in (E). 

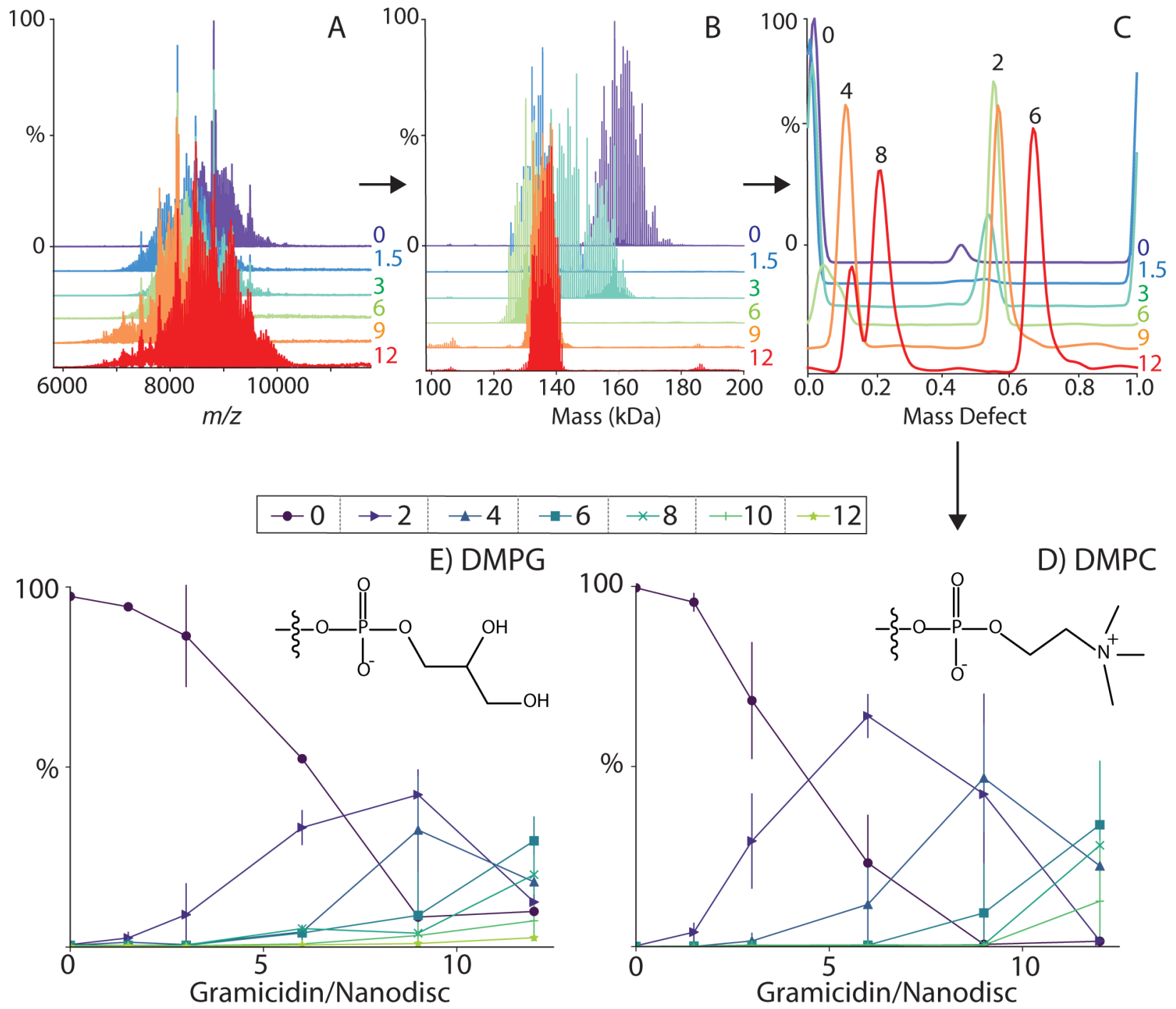

Figure 2.

Representative mass spectra (A), deconvolved mass distributions (B), and mass defects (C) for gramicidin A in DMPC nanodiscs with $25 \mathrm{mM}$ imidazole. Molar ratios of GA/nanodisc ranged from 0-12/1 and are labelled to right of spectra. Mass defect peaks in (C) are labelled with corresponding number of peptides incorporated. The relative intensities of different stoichiometries (0-12) of GA incorporated into DMPC (D) or DMPG (E) nanodiscs as function of the total GA/nanodisc molar ratio. Odd stoichiometries were not observed. 

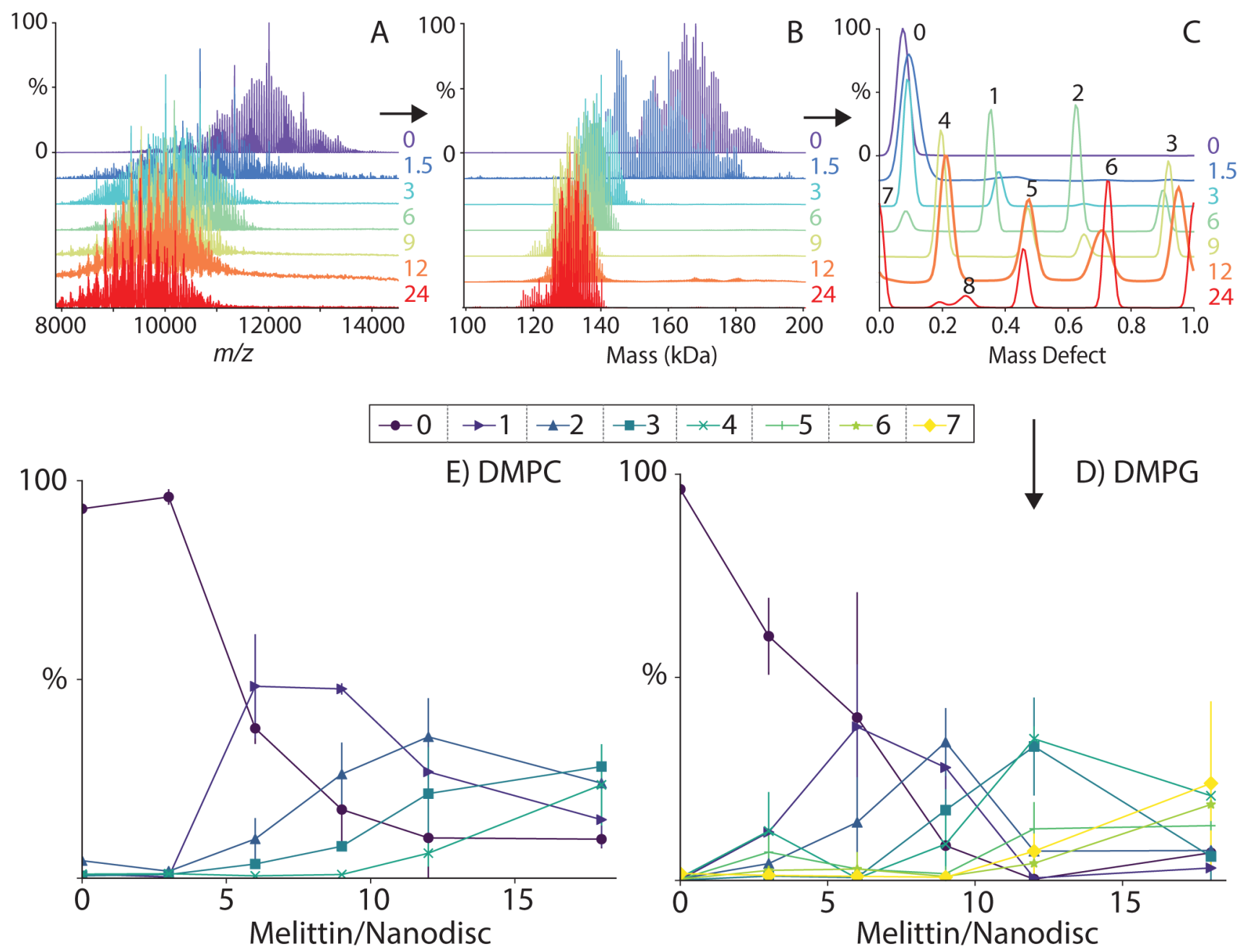

Figure 3.

Representative mass spectra (A), deconvolved mass distributions (B), and mass defects (C) for melittin in DMPG nanodiscs with $25 \mathrm{mM}$ imidazole. Molar ratios of melittin/nanodisc ranged from 0-24/1 and are labelled to right of spectra. Mass defect peaks in (C) are labelled with corresponding number of peptides incorporated. To assign mass defects, the predicted peptide mass defects (Table S1) are added to mass defect of 2 MSP belts (0.11). The relative intensities of different stoichiometries (0-18) of melittin incorporated into DMPG (D) or DMPC (E) nanodiscs as function of the total melittin/nanodisc molar ratio. 

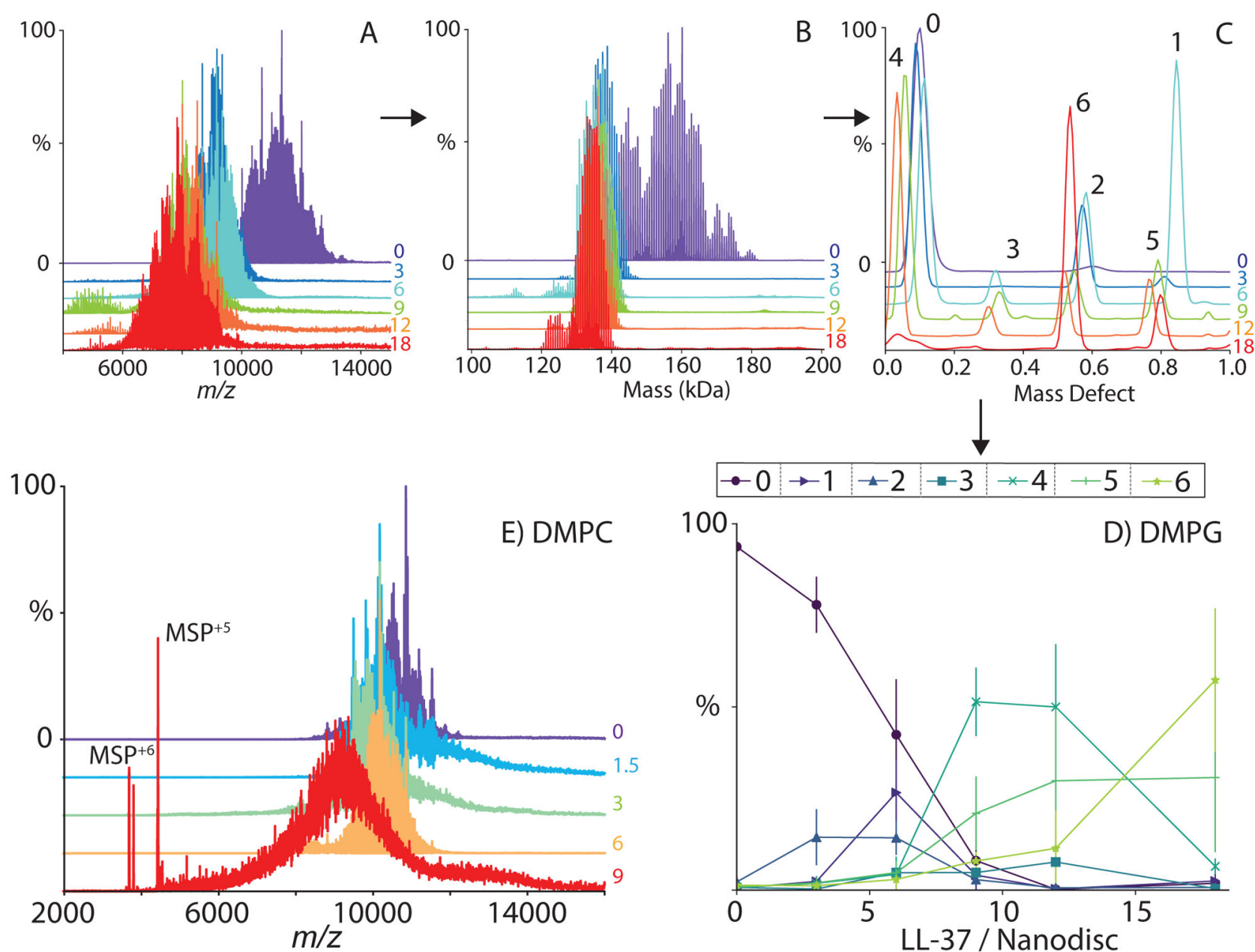

Figure 4.

Representative mass spectra (A), deconvolved mass distributions (B), and mass defects (C) for LL-37 in DMPG with $25 \mathrm{mM}$ imidazole. Molar ratios of LL-37/nanodisc ranged from 0-18 and are labelled to right of spectra. Mass defect peaks are labelled with corresponding number of peptides incorporated. To assign mass defects, the predicted peptide mass defects (Table S1) are added to mass defect of 2 MSP belts (0.11). The relative intensities of different stoichiometries (0-18) of LL-37 incorporated into DMPG (D) nanodiscs as function of the total LL-37/nanodisc molar ratio. Mass spectra of LL-37 titrated into DMPC (E) nanodiscs with molar ratios from 0-9 LL-37/nanodisc. At a 9/1 molar ratio, nanodiscs begin to dissociated and the main species detected were MSP belts. 